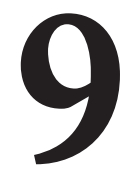

\title{
EL ESTUDIO DE LAS CREENCIAS SOBRE LA DIVERSIDAD CULTURAL COMO REFERENTE PARA LA MEJORA DE LA FORMACIÓN DOCENTE
}

\author{
(THE STUDY OF BELIEFS ABOUT CULTURAL DIVERSITY AS A REFERENCE \\ FOR IMPROVING TEACHER TRAINING)
}

\author{
M. ${ }^{a}$ del Carmen López López y Eva Fca. Hinojosa Pareja \\ Universidad de Granada
}

\section{RESUMEN}

En los últimos años han proliferado las propuestas dirigidas a la diversidad cultural sin que éstas hayan tenido el calado y efectividad que exige su naturaleza compleja. Los compromisos institucionales y personales, y con estos últimos las creencias, se convierten en foco de máximo interés. El sistema de creencias tiene un impacto en la práctica profesional, pudiendo representar un serio revés para una educación comprometida con la diversidad cultural. El estudio de las creencias en los futuros docentes se convierte así en objetivo central de este trabajo. A través de un estudio descriptivo por encuesta se profundiza en las creencias de estos docentes en torno a cinco dimensiones. Las conclusiones ponen de manifiesto, entre otras cosas, una concepción restrictiva de la diversidad cultural, una perspectiva poco crítica de la evaluación y la necesidad de una formación docente en y para la diversidad cultural.

\section{ABSTRACT}

In recent years proposals have proliferated to address cultural diversity but such initiatives haven't had the depth and effectiveness required by their complex nature. Institutional and personal commitments, and with the latter, also the beliefs, become a focus of great interest. The belief system has an impact on professional practice and may represent a serious setback for an education committed to cultural diversity. The study of beliefs in future teachers thus becomes the basic objective of this work. Through a descriptive study in a poll it 
deepens into beliefs of these professors in five key dimensions. Findings reveal, among other things, a restrictive view of cultural diversity, an uncritical perspective of evaluation and, most paramount of all, the need for teacher training in and for cultural diversity.

\section{ATENDER A LA DIVERSIDAD CULTURAL DESDE LA EDUCACIÓN. EL PAPEL DE LAS CREENCIAS Y LA FORMACIÓN DEL PROFESORADO}

Uno de los rasgos característicos de los centros educativos en estos momentos lo constituye la presencia de alumnado culturalmente diverso. Gestionar de forma adecuada tal diversidad y asegurar que la educación sea de calidad para todos, que promueva la construcción de una ciudadanía intercultural y democrática, es uno de los retos a los que se enfrenta el sistema educativo en general y el profesorado en particular (Ainscow, 2008). Responder a este desafío no resulta fácil en una escuela que no se ha mostrado especialmente sensible a este propósito. El etnocentrismo puesto de manifiesto, durante décadas, por los sistemas educativos ha conformado una institución educativa homogeneizadora y homogeneizada en cuanto a recursos, estrategias, contenidos y currículum en general, poco comprometida con la diversidad cultural, étnica, o de género (Dilworth \& Brown, 2001).

Atender a la diversidad cultural desde este escenario, como han señalado Abdallah-Pretceille (2001) y Besalú (2007), implica superar la noción actual de educación compensatoria y tender hacia la inclusión, un mayor compromiso con la equidad desde una educación de calidad para todos, instituciones educativas abiertas al entorno familiar y social, propuesta curriculares flexibles y consensuadas, y un profesorado convenientemente formado y comprometido con la diversidad cultural.

Reivindicaciones como estas son frecuentes en el campo de la bibliografía especializada, generalmente, teniendo como principales vectores la política educativa, el centro educativo y la cultura institucional, el currículum, y el profesorado. No obstante, las iniciativas emprendidas continúan teniendo un carácter puntual y sesgado, restringido a centros y voluntades concretas, sin que, por el momento, el cambio pretendido se haya hecho visible con carácter generalizado. La actual situación de transformación que experimentamos, que pone en entredicho los modelos sociales, políticos, económicos y educativos vigentes, puede ser una oportunidad para redefinir nuevos compromisos políticos e institucionales con la diversidad cultural, posibilitando que los sistemas educativos, los responsables de la formación docente y el propio profesorado se impliquen, de manera más decidida, en el pleno desarrollo de todos los ciudadanos. 
Contar con profesorado competente, capaz de sustentar su actividad y compromiso profesional en la profunda creencia de que todo el alumnado puede aprender, y en una ética profesional que busca el éxito de todos, es una garantía de calidad de cualquier sistema educativo (Escudero, 2006). La formación del profesorado es, en este sentido, un factor clave para educar en la diversidad cultural.

Formar buenos docentes no sólo implica dotarles del conocimiento necesario para desenvolverse con comodidad en un campo disciplinar, ni tan siquiera enseñarles a ser autónomos en su proceso de aprendizaje a lo largo de la vida, sino también, y fundamentalmente, promover unas actitudes, disposiciones y creencias determinadas (Gay, 2010; Korthagen, 2010). Desde esta perspectiva, formar EN y PARA la diversidad cultural comporta el desarrollo de propuestas que nos permitan disponer de un profesorado capaz de educar en contextos multiculturales con ciertas garantías de calidad, a la vez que se desarrollan como personas, ciudadanos y profesionales.

La formación del profesorado en y para la diversidad se encuentra en estos momentos en una situación paradójica, pues si bien es cierto que las investigaciones y contribuciones teóricas en este campo se ha incrementado sustancialmente, la práctica formativa continúa mostrándose infranqueable, haciendo que la formación del profesorado para la diversidad cultural sea inexistente o continúe recibiendo una atención marginal en los planes de estudios (Esteve, 2006), lo que explicaría que la forma en que se está abordando no esté resultando tan exitosa como cabría esperar, al menos en nuestro contexto.

Establecer compromisos sólidos, de naturaleza interdisciplinar y de carácter institucional, profesional y personal entre las instituciones responsables de la formación docente, los formadores y el propio profesorado continúa siendo una asignatura pendiente. La existencia de un amplio porcentaje de docentes poco favorables a la diversidad cultural representa, sin duda, un serio revés (Leiva, 2008; Rodríguez Izquierdo, 2010). Prestar atención a las creencias de los docentes se convierte así en un aspecto clave de la formación del profesorado. Las creencias hacia la diversidad cultural tienen especial influencia en la construcción del conocimiento del docente acerca de los alumnos, la enseñanza y en su propia práctica profesional (Shulman, 2005), ya que juegan un importante papel en la configuración de las expectativas sobre los estudiantes y éstas en sus aprendizajes.

La relevancia conferida a las creencias se incrementa en la formación inicial del profesorado, al ser uno de los periodos más críticos y decisivos en el proceso de aprender a enseñar, pues, en esta etapa, las creencias actúan como condicionantes del éxito o fracaso de este aprendizaje y del posterior 
desarrollo profesional del docente (Pajares, 1992; Van der Schaaf, Stokking \& Verloop, 2008). Este periodo formativo se convierte en un escenario ideal para favorecer su explicitación, convirtiéndolas en objeto de reflexión, e impulsando la construcción de un conocimiento más comprensivo, comprometido y sensible a la diversidad cultural en los futuros docentes.

Existen, en este sentido, distintos programas y propuestas que incluyen las creencias de los futuros docentes como referentes clave en la formación en y para la diversidad cultural (Gay, 2010; Sales Ciges, 2006). Algunos de los elementos incorporados a estas iniciativas contemplan la reflexión conjunta en torno a las creencias y la inclusión del componente comportamental y emocional en la formación, estableciendo nuevas relaciones entre lo cognitivo y afectivo (Grossman \& McDonald, 2008). Iniciativas como estas tienen como objetivo básico proporcionar experiencias significativas vinculadas a la diversidad cultural que permitan, a los futuros docentes, tomar conciencia de sus creencias y de la incidencia de estas en su práctica profesional, colocándoles en disposición de trascenderlas en pro de una finalidad altamente noble: la construcción de una sociedad más justa e inclusiva.

\section{ESTUDIO DE LAS CREENCIAS DE LOS FUTUROS DOCENTES ACERCA DE LA DIVERSIDAD CULTURAL}

\subsection{Objetivos}

El propósito de esta investigación es estudiar las creencias de los futuros docentes de Educación Infantil y Primaria de la Facultad de Ciencias de la Educación de la Universidad de Granada acerca de la diversidad cultural. Los objetivos que orientan este trabajo son

1. Elaborar un instrumento que permita recabar información sobre las creencias, respecto a la diversidad cultural, de los futuros profesores.

2. Estudiar las creencias del alumnado de magisterio de Educación Infantil y Primaria de la Facultad de Ciencias de la Educación de la Universidad de Granada.

\subsection{Metodología}

El interés por profundizar en lo que piensan los docentes, como medio para incrementar la comprensión acerca de los procesos educativos, surge como línea de investigación vinculada al paradigma de pensamiento del 
profesor hace algunas décadas. Desde esta línea de trabajo, nuestra investigación adopta un enfoque descriptivo (Cardona, 2005) desde el que pretendemos, a través de una aproximación cuantitativa mediante encuesta, describir las creencias sobre la diversidad cultural de los futuros docentes.

El cuestionario, escala tipo Likert, es de elaboración propia y está integrado por 79 ítems en forma de preguntas cerradas con cuatro opciones de respuesta. Se trata de un cuestionario politemático (Albert, 2007) en el que los ítems se estructuran en torno a cinco dimensiones:

- Sociedad culturalmente diversa: creencias acerca de la multiculturalidad como fenómeno social.

- Educación en contextos multiculturales: consideraciones relacionadas con la educación en contextos multiculturales.

- Profesorado: creencias relacionadas con el profesorado.

- Alumnado: creencias acerca del alumnado culturalmente heterogéneo.

- Práctica educativa: aspectos de naturaleza curricular presentes en la práctica formativa.

El cuestionario ha sido validado mediante revisión de jueces pertenecientes a distintas áreas de conocimiento con una media de 30 años de experiencia docente. La fiabilidad del mismo ha sido igualmente constatada a través de una prueba piloto y el posterior cálculo del Alfa de Cronbach, utilizando para ello el programa estadístico SPSS 15.0. El resultado obtenido es un coeficiente de $r=0,824$ con un nivel de confianza del $95 \%$ (p 0,05). Si entendemos que un rango de 0,600 se considera aceptable (Thorndike, 1997), podemos concluir que el cuestionario es altamente fiable. Hecho que queda ratificado al comprobar que los distintos coeficientes para cada ítem se sitúan entre 0,814 y 0,833 , lo que muestra que ningún ítem produce grandes variaciones en el contenido general del cuestionario y que todos, sin excepción, presentan una alta fiabilidad.

El instrumento ha sido aplicado a los estudiantes de Magisterio de Educación Infantil y Primaria de la Facultad de Ciencias de la Educación de la Universidad de Granada. Se trata de estudiantes con una edad comprendida, en su mayoría, entre los 18 y 22 años, con un alto porcentaje de representación femenina y de un nivel socio-económico medio.

El análisis de los datos obtenidos se basa en un análisis descriptivo básico. Los resultados que mostramos en este trabajo corresponden a los datos obtenidos con la aplicación de la prueba piloto del cuestionario. 


\section{RESULTADOS}

A continuación mostramos las puntuaciones más significativas (porcentajes, medias y desviaciones típicas), tras la aplicación del Inventario de creencias acerca de la diversidad cultural a los estudiantes de Magisterio de Educación Infantil y Primaria de Universidad de Granada, en cada una de las dimensiones de acuerdo con las opciones de respuesta: totalmente en desacuerdo (1), en desacuerdo (2), de acuerdo (3), y totalmente de acuerdo (4).

\subsection{Dimensión: Sociedad culturalmente diversa}

La primera dimensión (ítems 1-19) alude a la valoración que se hace de la diversidad cultural, su vinculación con las formas de organización sociopolítica y las desigualdades con las que puede asociarse. 


\begin{tabular}{|c|c|c|c|c|c|c|c|}
\hline \multirow{2}{*}{$\mathbf{N}^{\mathbf{o}}$} & \multirow{2}{*}{ Item } & \multicolumn{4}{|c|}{$\%$ respuestas } & \multirow{2}{*}{$\mathbf{X}$} & \multirow{2}{*}{ DT } \\
\hline & & 1 & 2 & 3 & 4 & & \\
\hline 1 & $\begin{array}{l}\text { La diversidad cultural enriquece la } \\
\text { convivencia ciudadana }\end{array}$ & 0,0 & 12,0 & 44,0 & 44,0 & 3,32 & ,683 \\
\hline 2 & $\begin{array}{l}\text { La Declaración de Derechos Humanos } \\
\text { constituye un referente válido para todas } \\
\text { las culturas }\end{array}$ & 0,0 & 16,3 & 55,1 & 28,6 & 3,12 & ,666 \\
\hline 4 & Todas las culturas poseen el mismo valor & 6,3 & 10,4 & 18,8 & 64,6 & 3,42 & 919 \\
\hline 5 & $\begin{array}{l}\text { Es el fenómeno la inmigración el que } \\
\text { confiere el carácter multicultural a los } \\
\text { contextos sociales }\end{array}$ & 0,0 & 17,8 & 73,3 & 8,9 & 2,91 &, 514 \\
\hline 6 & $\begin{array}{l}\text { La presencia de personas de culturas } \\
\text { distintas a la autóctona supone una } \\
\text { amenaza a la estabilidad social }\end{array}$ & 36,0 & 54,0 & 6,0 & 4,0 & 1,78 & ,737 \\
\hline 8 & $\begin{array}{l}\text { La existencia de unos valores compartidos } \\
\text { es imprescindible para la convivencia entre } \\
\text { culturas }\end{array}$ & 2,0 & 8,0 & 46,0 & 44,0 & 3,32 & ,713 \\
\hline 10 & $\begin{array}{l}\text { En sociedades plurales la mejor forma de } \\
\text { organización social es aquella que se } \\
\text { construye desde el diálogo entre las } \\
\text { distintas culturas existentes }\end{array}$ & 4,1 & 4,1 & 44,9 & 46,9 & 3,35 & ,751 \\
\hline 11 & $\begin{array}{l}\text { La diversidad de religiones y etnias es } \\
\text { un obstáculo para la convivencia en una } \\
\text { sociedad democrática }\end{array}$ & 46,0 & 34,0 & 20,0 & 0,0 & 1,74 & 777 \\
\hline 12 & $\begin{array}{l}\text { La política estatal debe definirse sobre } \\
\text { la base de los patrones de la cultura } \\
\text { mayoritaria }\end{array}$ & 6,4 & 40,0 & 38,3 & 14,9 & 2,62 & ,822 \\
\hline 16 & $\begin{array}{l}\text { El régimen democrático es el que } \\
\text { proporciona mayor desarrollo y } \\
\text { bienestar a todos los ciudadanos }\end{array}$ & 2,0 & 12,0 & 58,0 & 28,0 & 3,12 & 689 \\
\hline 19 & $\begin{array}{l}\text { Las culturas no autóctonas suelen estar } \\
\text { en una situación de desventaja social } \\
\text { respecto a la/s autóctona/s }\end{array}$ & 6,0 & 42,0 & 38,0 & 14,0 & 2,60 & 808, \\
\hline
\end{tabular}

Tabla 1. Dimensión: Sociedad culturalmente diversa

Si centramos nuestra atención en los porcentajes de respuesta más elevados encontramos dos ítems destacados, con porcentajes que superan el 60,0\%: el ítem 4, con un 64,6\% en la opción de respuesta «totalmente de acuerdo», y el ítem 5, con un $73,3 \%$ en la opción de respuesta "de acuerdo». 
Los mayores porcentajes globales de convergencia, superiores al 80\% en las opciones de respuesta «de acuerdo" $\mathrm{y}$ "totalmente de acuerdo», se encuentran en los ítems 1, 2, 4, 5, 8, 10 y 16. Especialmente relevantes los ítems 8 y 10, con porcentajes acumulados igual o superiores al 90\%. En la posición opuesta se encuentran los ítems 6 y 11, con porcentajes globales del 90 y $80 \%$ respectivamente en las opciones de respuesta «totalmente en desacuerdo»y «en desacuerdo».

Las discrepancias más acentuadas se aprecian en los ítems 12 y 19. En estos ítems las posiciones se encuentran divididas entre quienes se muestran "de acuerdo» y quienes se manifiestan claramente «en desacuerdo» con porcentajes muy similares (inferiores a 5 puntos) respecto a ambas posiciones, representando, de manera global, al 78 y $80 \%$ de los docentes del estudio respectivamente.

En cuanto a las puntuaciones medias más destacadas, encontramos el ítem 4 con la media más alta $(3,42)$ y el ítem 11 con la media más baja $(1,74)$.

Atendiendo a los valores alcanzados por las desviaciones típicas, observamos que el valor más bajo corresponde al ítem $5(0,514)$.

Especialmente relevantes son los ítems 1, 2 y 5 cuyas medias se corresponden con las puntuaciones más altas y sus desviaciones típicas con los valores más bajos, lo cual indica que los estudiantes de magisterio de Educación Infantil y Primaria que participan en la investigación se muestran especialmente de acuerdo con que la diversidad cultural enriquecen la convivencia, de Derechos Humanos es un referente válido para todas las culturas, y el fenómeno de la inmigración es el que confiere el carácter multicultural a las sociedades.

\subsection{Dimensión: La educación en contextos multiculturales}

La segunda dimensión (ítems 20-32) atiende a aspectos relacionados con los fines de la educación, los diferentes modelos de educación multicultural, el funcionamiento de los centros educativos en contextos multiculturales, la compensación educativa o la participación de las familias. 


\begin{tabular}{|c|c|c|c|c|c|c|c|}
\hline \multirow{2}{*}{$\mathbf{N}^{\mathbf{o}}$} & \multirow{2}{*}{ Item } & \multicolumn{4}{|c|}{$\%$ respuestas } & \multirow{2}{*}{$\mathbf{X}$} & \multirow{2}{*}{ DT } \\
\hline & & 1 & 2 & 3 & 4 & & \\
\hline 20 & $\begin{array}{l}\text { Una educación para todos debe aceptar } \\
\text { todas las formas culturales existentes }\end{array}$ & 4,0 & 4,0 & 36,0 & 56,0 & 3,44 & ,760 \\
\hline 21 & $\begin{array}{l}\text { Educar en la diversidad cultural sólo es } \\
\text { necesario cuando hay alumnado de otros } \\
\text { países }\end{array}$ & 48,0 & 40,0 & 8,0 & 4,0 & 1,68 & ,794 \\
\hline 22 & $\begin{array}{l}\text { La formación religiosa de los estudiantes } \\
\text { es competencia de la escuela }\end{array}$ & 34,7 & 42,9 & 14,3 & 8,2 & 1,96 & ,912 \\
\hline 23 & $\begin{array}{l}\text { El sistema educativo debe generar medidas } \\
\text { que compensen las posibles desigualdades } \\
\text { por razones de origen cultural }\end{array}$ & 2,0 & 10,0 & 58,0 & 30,0 & 3,16 & ,681 \\
\hline 24 & $\begin{array}{l}\text { La institución escolar debe favorecer el } \\
\text { aprendizaje de unos valores éticos } \\
\text { compartidos }\end{array}$ & 2,0 & 6,0 & 48,0 & 44,0 & 3,34 & 688 \\
\hline 26 & $\begin{array}{l}\text { La educación reproduce y transmite } \\
\text { prioritariamente los patrones culturales } \\
\text { del grupo social hegemónico }\end{array}$ & 6,3 & 37,5 & 50,0 & 6,3 & 2,56 & ,712 \\
\hline 28 & $\begin{array}{l}\text { El funcionamiento de los centros } \\
\text { educativos en contextos multiculturales } \\
\text { responde a una dinámica participativa }\end{array}$ & 4,1 & 18,4 & 59,2 & 18,4 & 2,92 & ,731 \\
\hline 32 & $\begin{array}{l}\text { Los centros educativos que acogen } \\
\text { alumnado de distintas culturas tienen } \\
\text { menos prestigio social }\end{array}$ & 34,0 & 40,0 & 20,0 & 6,0 & 1,98 & ,892 \\
\hline
\end{tabular}

Tabla 2. Dimensión: La educación en contextos multiculturales

De acuerdo con los porcentajes de respuesta obtenidos, destacan los ítems 20, 23 y 28, todos ellos con puntuaciones superiores al 55\% en las opciones de respuesta «de acuerdo» $\mathrm{o}$ «totalmente de acuerdo».

Los porcentajes más elevados de convergencia en las posiciones «de acuerdo» o "totalmente de acuerdo» corresponden a los ítems 20 y 24, con un porcentaje acumulado del $92 \%$, y el ítem 22 , cuyo porcentaje acumulado es del $88 \%$. En el extremo opuesto se encuentran los ítems 21, 22 y 32, con porcentajes globales del 88, 77 y $74 \%$ respectivamente en las opciones de respuesta «totalmente en desacuerdo» $\mathrm{y}$ «en desacuerdo».

El mayor desencuentro se aprecia en el ítem 26. En este ítem el 87,5\% de los estudiantes de magisterio se posicionan de manera equilibrada entre las opción «de acuerdo» (50\%) y «en desacuerdo» (37.5\%), evidenciando así la discrepancia de sus creencias en este punto. 
En relación a las medias, comprobamos que los valores más altos corresponden a los ítems $20(3,44)$ y $24(3,34)$, mientras que las medias más bajas se encuentran en el ítem $21(1,68)$ y $22(1.96)$.

Centrándonos en los valores alcanzados por las desviaciones típicas, las puntuaciones más bajas corresponden a los ítems $23(0,681)$ y $24(0,688)$.

Finalmente, destacamos un conjunto de ítems que, por los valores elevados de sus medias y las puntuaciones bajas alcanzadas en la desviación típica, evidencian un gran nivel de acuerdo entre los futuros docentes. Nos referimos a los ítems 20, 23 y 24. Esta constatación nos lleva a afirmar que los alumnos creen, mayoritariamente, que la educación para todos debe aceptar todas las manifestaciones culturales, que el sistema educativo está obligado a generar medidas que compensen las posibles desigualdades por motivos culturales, y que la institución educativa debe favorecer el aprendizaje de valores éticos compartidos por todos.

\subsection{Dimensión: Profesorado}

La tercera dimensión (ítems 33-54) recoge las creencias acerca del profesorado, su formación para el desempeño profesional en contextos multiculturales y su rol en función de los distintos modelos de educación multicultural, entre otras. 


\begin{tabular}{|c|c|c|c|c|c|c|c|}
\hline \multirow{2}{*}{$\mathbf{N}^{\mathbf{o}}$} & \multirow{2}{*}{ Item } & \multicolumn{4}{|c|}{$\%$ respuestas } & \multirow{2}{*}{$\mathbf{X}$} & \multirow{2}{*}{ DT } \\
\hline & & 1 & 2 & 3 & 4 & & \\
\hline 33 & $\begin{array}{l}\text { El profesorado está cualificado para } \\
\text { desarrollar su labor docente en aulas } \\
\text { culturalmente heterogéneas }\end{array}$ & 4,0 & 56,0 & 26,0 & 14,0 & 2,50 & ,789 \\
\hline 35 & $\begin{array}{l}\text { El modelo educativo que se propone con } \\
\text { el Espacio Europeo de Educación } \\
\text { Superior mejorará la formación del } \\
\text { profesorado para la diversidad cultural }\end{array}$ & 2,1 & 21,3 & 57,4 & 19,1 & 2,94 & ,704 \\
\hline 38 & $\begin{array}{l}\text { Sólo los profesores que trabajan con } \\
\text { alumnado de distintas culturas deben } \\
\text { tener una formación específica para } \\
\text { atender a la diversidad cultural }\end{array}$ & 46,0 & 44,0 & 8,0 & 2,0 & 1,66 & ,717 \\
\hline 39 & $\begin{array}{l}\text { Las creencias positivas del profesorado } \\
\text { hacia la diversidad cultural del alumnado } \\
\text { influyen de manera favorable en el } \\
\text { desempeño de su actividad profesional }\end{array}$ & 0,0 & 20 & 50 & 30 & 3,10 & ,707 \\
\hline 41 & $\begin{array}{l}\text { La mayoría del profesorado en ejercicio } \\
\text { tiene prejuicios respecto al alumnado } \\
\text { procedente de culturas distintas a la propia }\end{array}$ & 16,0 & 36,0 & 42,0 & 6,0 & 2,38 & ,830 \\
\hline 45 & $\begin{array}{l}\text { La formación para atender a la diversidad } \\
\text { cultural se adquiere durante el ejercicio } \\
\text { profesional }\end{array}$ & 6,0 & 36,0 & 46,0 & 12,0 & 2,64 & ,776 \\
\hline 47 & $\begin{array}{l}\text { La colaboración entre profesores es } \\
\text { imprescindible para el trabajo en aulas } \\
\text { multiculturales }\end{array}$ & 4,0 & 6,0 & 44,0 & 46,0 & 3,32 & ,768 \\
\hline 51 & $\begin{array}{l}\text { Durante el periodo de formación inicial } \\
\text { del profesorado debe haber asignaturas } \\
\text { específicas que preparen para trabajar } \\
\text { con colectivos culturalmente diversos }\end{array}$ & 4,0 & 14,0 & 54,0 & 28,0 & 3,06 & ,767 \\
\hline 53 & $\begin{array}{l}\text { Las creencias de los docentes respecto a } \\
\text { la diversidad cultural no se modifican con } \\
\text { la formación inicial que reciben en la } \\
\text { universidad }\end{array}$ & 4,3 & 38,3 & 48,9 & 8,5 & 2,62 & ,709 \\
\hline 54 & $\begin{array}{l}\text { La formación del profesorado para la } \\
\text { diversidad cultural es innecesaria }\end{array}$ & 56,0 & 36,0 & 8,0 & 0,0 & 1,52 & ,646 \\
\hline
\end{tabular}

Tabla 3. Dimensión: Profesorado

En este caso, los porcentajes de respuesta más elevados, superiores al $55 \%$, corresponden a los ítems 33, 35, y 54 . 
La concentración más destacada de porcentajes, igual o superior al $80 \%$, en las opciones de respuesta «de acuerdo» y «totalmente de acuerdo» se encuentran en los ítems 39 (80\%), 47 (90\%) y 51 (82\%). En el caso opuesto se encuentran los ítems 38 y 54 cuyos porcentajes globales de respuesta en las opciones «totalmente en desacuerdo» $\mathrm{y}$ «en desacuerdo» alcanza el $90 \mathrm{y}$ $92 \%$ respectivamente.

Las discrepancias más acentuadas entre los estudiantes de magisterio de Educación Infantil y Educación Primaria se aprecian en los ítems 41, 45 y 53. En estos ítems las posiciones se encuentran divididas entre quienes se muestran "de acuerdo" y quienes se manifiestan claramente "en desacuerdo» con porcentajes muy equilibrados respecto a ambas posiciones, representando, de manera global, al 78, 82 y $87,2 \%$ de los docentes del estudio respectivamente.

En relación a las medias, los ítems más destacados son el 39 (3.10) y el 47 (3.32) con las medias más altas. Los valores más bajos corresponden a los ítems $38(1.66)$ y 54 (1.52).

Si nos detenemos un momento en los valores obtenidos en la desviación típica, observamos que las puntuaciones más bajas $(0,704,0,707$ y 0,646) corresponden a los ítems 35, 39 y 54.

Especial mención merecen los ítems 39, 47 y 54. En el caso de los dos primeros, encontramos unas altas puntuaciones medias junto a unas desviaciones típicas especialmente bajas, lo cual muestra que los estudiantes de Magisterio de Infantil y Primaria tienden a considerar que las creencias positivas de los docentes influyen favorablemente en su desempeño profesional y que la colaboración entre el profesorado es imprescindible cuando se trabaja en aulas multiculturales. El ítem 54, por su parte, presenta la menor media y desviación típica de la tabla, lo que indica que la mayoría de los futuros docentes se muestran totalmente en desacuerdo con que la formación del profesorado para la diversidad cultural pueda ser considerada innecesaria.

\subsection{Dimensión: Alumnado}

Esta cuarta dimensión (ítems 55-67) se centra en cuestiones como: agrupamientos, situación y consideración del alumnado culturalmente heterogéneo en el centro educativo, dificultades de aprendizaje y fracaso escolar. 


\begin{tabular}{|l|l|c|c|c|c|c|c|}
\hline \multirow{2}{*}{$\mathbf{N}^{\mathbf{0}}$} & \multicolumn{3}{|c|}{ Item } & \multirow{2}{*}{ X } & \multirow{2}{*}{ DT } \\
\cline { 3 - 6 } & $\mathbf{1}$ & $\mathbf{2}$ & $\mathbf{3}$ & $\mathbf{4}$ & & \\
\hline 55 & $\begin{array}{l}\text { La presencia de alumnado de diferentes } \\
\text { culturas enriquece la institución educativa }\end{array}$ & 0,0 & 18,0 & 46,0 & 36,0 & 3,18 &, 720 \\
\hline 56 & $\begin{array}{l}\text { La presencia de estudiantes de culturas } \\
\text { distintas a la autóctona es más patente en } \\
\text { centros públicos que en privados y/o } \\
\text { concertados }\end{array}$ & 6,3 & 16,7 & 45,8 & 31,1 & 3,02 &, 863 \\
\hline 58 & $\begin{array}{l}\text { Para el buen funcionamiento del aula es } \\
\text { necesario que los estudiantes se agrupen } \\
\text { según su cultura de procedencia }\end{array}$ & 54,0 & 38,0 & 6,0 & 2,0 & 1,56 &, 705 \\
\hline 59 & $\begin{array}{l}\text { La cultura de origen del alumnado no } \\
\text { autóctono es causa de muchos de los } \\
\text { problemas de convivencia en la escuela }\end{array}$ & 16,3 & 44,9 & 38,8 & 0,0 & 2,22 &, 715 \\
\hline 62 & $\begin{array}{l}\text { El alumnado culturalmente diverso recibe } \\
\text { apoyos específicos para incorporarse en } \\
\text { igualdad de condiciones a los centros } \\
\text { educativos }\end{array}$ & 0,0 & 42,9 & 51,0 & 6,1 & 2,63 &, 602 \\
\hline 63 & $\begin{array}{l}\text { La mayor parte del alumnado de culturas } \\
\text { distintas a la autóctona proviene de } \\
\text { contextos marginales }\end{array}$ & 8,2 & 40,8 & 42,9 & 8,2 & 2,51 &, 767 \\
\hline 64 & $\begin{array}{l}\text { Los estudiantes de procedencia cultural } \\
\text { distinta a la autóctona presentan altos } \\
\text { niveles de fracaso escolar }\end{array}$ & 6,3 & 54,2 & 27,1 & 12,5 & 2,46 &, 798 \\
\hline 66 & $\begin{array}{l}\text { El alumnado de diferentes culturas } \\
\text { muestra dificultad para integrarse } \\
\text { socialmente }\end{array}$ & 8,3 & 37,5 & 43,8 & 10,4 & 2,56 &, 796 \\
\hline 67 & $\begin{array}{l}\text { La presencia de alumnado culturalmente } \\
\text { heterogéneo en el aula favorece el } \\
\text { aprendizaje }\end{array}$ & 0,0 & 20,4 & 67,3 & 12,2 & 2,92 &, 571 \\
\hline
\end{tabular}

Tabla 4. Dimensión: Alumnado

Si nos centramos en los porcentajes de respuesta alcanzados por los ítems de esta dimensión, observamos que son tres los ítems cuya puntuación es igual o superior al 54\%. Nos referimos a los ítems $58(54 \%), 64(54,2 \%)$ y $67(67,3 \%)$.

Las tendencias más destacas en cuanto a los niveles de acuerdo se aprecian en los ítems 55 (82\%), $56(76.9 \%)$ y 67 (79.5\%) que concentran porcentajes globales superiores al $75 \%$ en la opciones de respuesta «de acuerdo» 
o «totalmente de acuerdo». El porcentaje acumulado en las opciones de respuesta «totalmente en desacuerdo» $\mathrm{o}$ "en desacuerdo» es especialmente significativo en el ítem 58 (92\%).

Los mayores desencuentros se producen en los ítems 59, 62, 63 y 66 . En todos ellos las posiciones se polarizan de manera equilibrada (inferior a 10 puntos), con porcentajes globales que superan el 80\%, entre la opción de respuesta «en desacuerdo» $\mathrm{y}$ «de acuerdo».

Atendiendo a los valores alcanzados por las medias, encontramos que los valores más altos corresponden a los ítems 55 (3.18) y 56 (3.02). La media más baja pertenece al ítem $58(1,56)$.

En relación a las desviaciones típicas, observamos que la puntuación más baja, corresponde al ítem $67(0,571)$.

Para finalizar destacamos los ítems 58 y 67, ambos con valores bajos en la desviación típica y altos en la media. Esta constatación nos permite declarar que los estudiantes de Magisterio de Educación Infantil y Primaria están mayoritariamente en desacuerdo con que el agrupamiento del alumnado se realice en función de su procedencia cultural, y ampliamente de acuerdo con que la presencia de alumnado culturalmente diverso favorece el aprendizaje.

\subsection{Dimensión: Práctica Educativa}

Esta última dimensión (ítems 68-79) centra su atención en cuestiones estrictamente curriculares como planificación, contenidos, objetivos, clima del aula, evaluación, recursos y la propia acción docente. 


\begin{tabular}{|c|c|c|c|c|c|c|c|}
\hline \multirow{2}{*}{$\mathbf{N}^{\mathbf{o}}$} & \multirow{2}{*}{ Item } & \multicolumn{4}{|c|}{$\%$ respuestas } & \multirow{2}{*}{$\mathbf{X}$} & \multirow{2}{*}{ DT } \\
\hline & & 1 & 2 & 3 & 4 & & \\
\hline 68 & $\begin{array}{l}\text { Los estudiantes suelen mostrar reticencias } \\
\text { a relacionarse con alumnado de grupos } \\
\text { culturales distintos al propio }\end{array}$ & 6,1 & 40,8 & 44,9 & 8,2 & 2,55 & ,738 \\
\hline 69 & $\begin{array}{l}\text { Las actuales propuestas curriculares se } \\
\text { adaptan a las diferencias culturales } \\
\text { presentes en el aula }\end{array}$ & 2,0 & 46,9 & 42,9 & 8,2 & 2,57 & 677 \\
\hline 70 & $\begin{array}{l}\text { La cuestión básica a la que el profesor debe } \\
\text { prestar atención en aulas culturalmente } \\
\text { heterogéneas es el aprendizaje de la lengua }\end{array}$ & 4,1 & 42,9 & 44,9 & 8,2 & 2,57 & ,707 \\
\hline 71 & $\begin{array}{l}\text { Los contenidos curriculares contemplan } \\
\text { los valores de las diferentes culturas } \\
\text { representadas en el aula }\end{array}$ & 6,5 & 45,7 & 45,7 & 2,2 & 2,43 & ,655 \\
\hline 72 & $\begin{array}{l}\text { La evaluación en aulas multiculturales se } \\
\text { apoya en estrategias diversas para } \\
\text { adaptarse a las peculiaridades culturales } \\
\text { de cada estudiante }\end{array}$ & 4,3 & 48,9 & 36,2 & 10,6 & 2,53 & ,747 \\
\hline 73 & $\begin{array}{l}\text { Los libros de texto reproducen estereotipos } \\
\text { culturales }\end{array}$ & 6,1 & 28,6 & 57,1 & 8,2 & 2,67 & ,718 \\
\hline 74 & $\begin{array}{l}\text { La planificación curricular responde a } \\
\text { criterios basados principalmente en las } \\
\text { singularidades de la cultura mayoritaria }\end{array}$ & 2,1 & 36,2 & 46,8 & 14,9 & 2,74 & ,736 \\
\hline 77 & $\begin{array}{l}\text { Los docentes deben centrar su enseñanza } \\
\text { en las diferencias culturales de los } \\
\text { estudiantes }\end{array}$ & 10,4 & 43,8 & 37,5 & 8,3 & 2,44 & ,796 \\
\hline 79 & $\begin{array}{l}\text { El sistema de evaluación utilizado } \\
\text { actualmente perjudica al alumnado de } \\
\text { culturas distintas a la autóctona }\end{array}$ & 6,3 & 50,0 & 39,6 & 4,2 & 2,42 & ,679 \\
\hline
\end{tabular}

Tabla 5. Dimensión: Práctica educativa

Una breve mirada a los valores alcanzados evidencia, lo que quizá sea el aspecto más destacable de la misma, la división y el desencuentro en las creencias de los futuros docentes cuando se abordan cuestiones relativas a la práctica educativa en contextos multiculturales.

Si atendemos a los porcentajes, encontramos que sólo dos ítems presentan puntuaciones iguales o superiores al 50\%. Nos referimos a los ítems $73(57,1 \%)$ y $79(50 \%)$. 
Los porcentajes más elevados de convergencia en las posiciones «de acuerdo» $\mathrm{y}$ «totalmente de acuerdo» corresponden a los ítems 73 y 74 con un porcentaje acumulado superior al 60\%. En el extremo opuesto se encuentran los ítems 72, 77 y 79 con porcentajes globales superiores al 50\% en las opciones de respuesta «totalmente en desacuerdo» $\mathrm{y}$ «en desacuerdo».

Las discrepancias más acentuadas respecto a la práctica profesional se aprecian en los ítems 68, 69, 70 y 71. En estos ítems las posiciones se encuentran divididas entre quienes se muestran «en desacuerdo» y quienes se manifiestan claramente "de acuerdo" con porcentajes muy cercanos en ambas posiciones (inferiores a 5 puntos), y con un porcentaje global superior al 85\% de las respuestas. Especial mención merece el ítem 71, donde el porcentaje de respuesta es de $45.7 \%$ para las dos opciones de respuesta señaladas.

Centrándonos en los valores alcanzados por las medias, se aprecia que oscilan entre 2,42 (ítem 79) y 2,74 (ítem 74).

En el caso de la desviación típica, encontramos una alta concentración de las puntuaciones en torno a la media. El valor más bajo corresponde al ítem 71 .

Especialmente significativos resultan los ítems 71, 73, 74 y 79. Así, el ítem 71, con la desviación típica más baja y con un porcentaje global de respuestas del 91.4\%, repartido equitativamente entre las opciones «en desacuerdo» $\mathrm{y}$ «de acuerdo», evidencia que la mayor discrepancia entre los futuros docentes se produce al considerar que los contenidos curriculares contemplan los valores de las diferentes culturas representadas en el aula. Más allá de esta divergencia, observamos, en relación a los ítems 73 y 74, que los estudiantes de Magisterio de Educación Infantil y Educación Primaria se muestran especialmente convencidos de que los libros de texto reproducen estereotipos culturales, y que la planificación curricular responde a criterios basados principalmente en las peculiaridades de la cultura mayoritaria. Finalmente, el ítem 79, con la media y desviación típica más bajas de la tabla y un porcentaje acumulado superior al 55\% en la opción «totalmente en desacuerdo» y "en desacuerdo», pone de manifiesto que la consideración de que el sistema actual de evaluación perjudica al alumno de culturas distintas es el aspecto con el que más en desacuerdo se manifiestan los futuros docentes.

\section{DISCUSIÓN DE RESULTADOS Y CONCLUSIONES}

Los resultados obtenidos evidencian que los futuros docentes creen, mayoritariamente, que el fenómeno migratorio es el que confiere el carácter 
multicultural a la sociedad actual, desconsiderando así otros factores vinculados al hecho multicultural y la naturaleza, culturalmente diversa, de los propios grupos e individuos. La formación del profesorado debería promover procesos de análisis más globales y complejos e incorporar los avances en el campo de la investigación antropológica. Estos avances subrayan el carácter dinámico de la propia cultura y su consideración como producto resultante de la comunicación, intercambio y transacciones que acontecen, tanto en el seno de los propios grupos, como en las interacciones entre ellos.

Aunque tienden a considerar que todas las culturas tiene el mismo valor, sus posiciones divergen sustancialmente cuando se trata de clarificar el peso que la cultura mayoritaria debe jugar en la definición de la política a seguir, o la situación de desigualdad social que puedan experimentar los miembros de culturas distintas a la autóctona. En este sentido, los futuros docentes creen que la valoración igualitaria, en esencia, de las distintas culturas, no implica necesariamente una participación igualitaria y funcional en la organización de la vida social, que parece quedar supeditada a otros factores como el hecho de ser grupo mayoritario, cuestión esta ya abordada en el famoso ensayo de Taylor (1993), y que, desde la perspectiva de los estudiantes de Magisterio, no tiene necesariamente que traducirse en situaciones de desventaja social. El privilegio conferido a los patrones de la cultura mayoría en la política y la creencia de que esto no se traduce en una situación de desventaja social para el resto de culturas, pone de manifiesto la necesidad de una formación política y moral del profesorado que les ayude a apreciar las interacciones existentes entre las diferentes dimensiones de la realidad (política, social, educativa,...) e incremente su compromiso con la igualdad social como sugieren Zeichner (2009).

Los aspirantes a profesores consideran que la diversidad cultural no supone una amenaza a la estabilidad social, ni un obstáculo para la convivencia, más bien creen que la enriquece, y que la mejor forma de organización social es aquella que se construye desde el diálogo, un diálogo que debe conducir a la adopción de unos valores compartidos, necesarios para garantizar la convivencia. Coinciden, así, con Adela Cortina (2010) cuando afirma que el diálogo entre las distintas culturas debe asentarse en una ética intercultural que permita descubrir, conjuntamente, qué aportaciones resultan verdaderamente valiosas y cuáles no para garantizar una convivencia más justa. En este sentido, los futuros docentes consideran que la Declaración de Derechos Humanos es un punto de partida válido.

Los estudiantes de Educación Infantil y Primaria creen, igualmente, que una educación para todos debe aceptar todas las formas culturales y que el sistema educativo debe adoptar medidas que compensen las desigualdades derivadas del origen cultural. La primera de estas apreciaciones, propia de la 
postmodernidad, denota una falta de reflexión crítica en torno a las diferencias culturales que no son legítimas, ni respetables, bien porque atentan a la propia dignidad humana, o bien porque se desarrollan al margen del orden legal establecido. No se aprecia una toma de conciencia generalizada en relación a que hay formas culturales inaceptables, no porque no se compartan, como señala Cortina (1999), sino porque son inmorales. Esta constatación pone de manifiesto la necesidad de emprender acciones formativas que promuevan procesos de deliberación acerca de las diferencias que son respetables y aquellas otras que no lo son, confiriendo así un peso específico importante a la formación moral del profesorado desde la propia formación inicial.

La consideración de que la diversidad cultural debe dirigirse a todo el alumnado, y no sólo a los que proceden de otros países, pone de manifiesto que sus creencias se alejan de viejas concepciones que vinculan la educación para la diversidad cultural a contextos específicos en los que esta diversidad se hace especialmente constatable.

Los futuros docentes difieren al considerar que la educación actúa como mecanismo reproductor de los patrones culturales del grupo hegemónico. En este sentido se aprecia una división de opiniones que evidencia la desconsideración, por parte de un número importante de estudiantes, de la carga ideológica y política que acompaña a la educación y de su función en los procesos de reproducción social (Giroux, 2004). Esta evidencia pone de manifiesto el impacto de los enfoques, básicamente transmisores, en los que se ha sustentado la formación y la falta de compromiso de las instituciones educativas con el desarrollo de la pedagogía crítica.

La mayoría de los futuros docentes creen que los profesores no están cualificados para trabajar en aulas multiculturales, aspecto este subrayado, entre otros, por Tedesco (2001) y López (2002). Consideran necesario emprender acciones dirigidas a formar al profesorado en la diversidad cultural, creen que la incorporación de materias específicas en la formación inicial del profesorado es necesaria y opinan que la formación para la diversidad debe ir dirigida a todo el profesorado, y no sólo a los docentes que trabajan con alumnado culturalmente diverso. Esta relevancia conferida a la formación docente choca, sin embargo, con las discrepancias manifestadas al considerar que las creencias respecto a la diversidad cultural no se modifican con la formación inicial, aspecto este denunciado por Pajares (1992) y, más tarde, por Marcelo (2009).

Los futuros docentes piensan, mayoritariamente, que las creencias positivas hacia la diversidad cultural del profesorado influyen favorablemente en la práctica docente, reconociendo así la importancia de las creen- 
cias y su impacto en la enseñanza y el aprendizaje. Discrepan, sin embargo, al considerar que la mayoría del profesorado en ejercicio tiene prejuicios respecto al alumno procedente de otras culturas, lo que implica que un número importante de futuros docentes cree que muchos profesores no tienen una disposición favorable hacia la diversidad cultural, confirmando los hallazgos de Leiva (2008) y Gay (2010). Quizá por ello, los estudiantes de Educación Infantil y Primaria piensan que lo mejor es afrontar la educación en contextos multiculturales desde la colaboración docente, compartiendo así la importancia conferida a ésta en los nuevos enfoques de la formación del profesorado.

Los aspirantes a profesores que participan en el estudio creen que la presencia de alumnado culturalmente diverso enriquece la institución educativa y favorece el aprendizaje. La mayor parte de ellos comparten la idea de que la presencia de alumnado culturalmente diverso es más patente en los centros públicos que en los privados y concertados, aspecto ratificado por las estadísticas de escolarización. Los estudiantes de Magisterio se muestran, sin embargo, claramente contrarios al agrupamiento del alumnado en función de su procedencia cultural y no creen que el alumnado de culturas distintas a la autóctona presente altos niveles de fracaso escolar. Esta última creencia les aleja de enfoques que centran el objetivo de la educación para la diversidad cultural en la compensación de los déficits (reales o supuestos) de las culturas distintas a la mayoritaria.

El desencuentro entre las posiciones referidas al alumnado de otras culturas se produce cuando se plantea el tipo de contextos de los que proceden, si la cultura de origen del alumnado es la causa de los problemas de convivencia en los centros, si el alumnado de diferentes culturas muestra dificultades para integrarse socialmente, o si reciben los apoyos que necesitan para incorporarse, en igualdad de condiciones, a los centros educativos. En estos casos las consideraciones discrepan sustancialmente no apreciándose ninguna tendencia clara.

El estudio realizado evidencia que las mayores discrepancias se producen cuando se abordan temas estrechamente vinculados con la práctica educativa. En estos casos las posiciones tienden a polarizarse, quizá por el abismo existente entre la declaración de principios relativos a la diversidad cultural, lo políticamente correcto, y lo que, en coherencia, implicaría su proyección a la práctica formativa.

En este punto, los aspirantes a docentes creen que los libros de texto reproducen estereotipos culturales, la planificación curricular se apoya en las singularidades de la cultura mayoritaria, y las estrategias de evaluación no son diversificadas para adaptarse a las peculiaridades culturales de los es- 
tudiantes, lo que confirma la falta de compromiso con la diversidad cultural (Dilworth \& Brown, 2001). Lo paradójico de esta situación es que, a pesar de estas declaraciones, los futuros docentes no creen que el actual sistema de evaluación perjudique al alumnado culturalmente diverso. Esta constatación evidencia la perspectiva poco crítica desde la que los estudiantes de magisterio analizan y valoran la práctica educativa en la formación docente.

Los desencuentros más destacados entre los futuros docentes, en relación con la práctica, se producen cuando se plantea que las actuales propuestas curriculares no se adaptan a la diversidad cultural del aula y los contenidos que se proponen no contemplan los valores de las diferentes culturas. En sendos aspectos las posiciones tienden a polarizarse especialmente entre quienes se muestran de acuerdo y en desacuerdo, dejando al descubierto que existe un número importante de estudiantes de Magisterio que creen que no se planifica, ni se seleccionan los contenidos atendiendo a la diversidad cultural del aula. 


\section{REFERENCIAS BIBLIOGRÁFICAS}

Abdallah-Pretceille, M. (2001). La educación intercultural. Barcelona: Idea Books.

Ainscow, M. (2008). Garantizar que cada alumno es importante: la mejora de la equidad dentro de los sistemas, en Gairín, J. \& Antúnez, S. (ed.) Organizaciones educativas al servicio de la sociedad. Madrid: Wolters Kluwer, 261-282.

Albert, M. J. (2007). La investigación educativa. Claves teóricas. Madrid: McGraw Hill.

Besalú, X. (2007). Educar en sociedades pluriculturales. Barcelona: Wolters Kluwer.

Cardona, C. (2005). Diversidad y Educación Inclusiva: Enfoques metodológicos y estrategias para una enseñanza colaborativa. Madrid: Pearson.

Cortina, A. (1999). Ciudadanos del mundo. Hacia una teoría de la ciudadanía. Madrid: Alianza Popular.

Cortina, A. (2010). Justicia cordial. Madrid: Trotta.

Dilworth, M. E. \& Brown, C. E. (2001) Considerer the difference: teaching and learning in culturally rich schools, en Richardson V. Handbook of research on teaching. Washington: AERA, 643667.

Escudero, J. M. (2006). La formación del profesorado y la garantía del derecho a una buena educación para todos, en J. Escudero, M., \& Gómez, A. L., (edits.) La formación del profesorado y la mejora de la educación. Barcelona: Octaedro, 21-51.

Esteve, J. M. (2006). La profesión docente en Europa: perfil, tendencias y problemática. La formación inicial. Revista de Educación, 340, 19-40.
Gay, G. (2010). Acting on beliefs in Teacher Education for Cultural Diversity. Journal of Teacher Education, 61 (1-2), 143-152.

Giroux, H. A. (2004). Teoría y resistencia en educación: una pedagogía para la oposición. México: Siglo XXI.

Grossman, P. \& McDonald, M. (2008). Back to the future: directions for research in teaching and teacher education. American Educational Research Journal, 45 (1), 184-205.

Korthagen, F. A. (2010). La práctica, la teoría y la persona en la formación del profesorado. Revista Interuniversitaria de Formación del Profesorado, 68 (24, 2), 83-102.

Leiva, J. J. (2008). Interculturalidad, gestión de la convivencia y diversidad cultural en la escuela: un estudio de las actitudes del profesorado. Revista Iberoamericana de Educación, 46 (2), 114.

López López, M. C. (2002). Diversidad socio-cultural y formación de profesores. Bilbao: Mensajero.

Marcelo, C. (2009). El profesorado principiante. Inserción a la docencia. Barcelona: Octaedro.

Pajares, M. F. (1992). Teachers Beliefs and Educational Research: Cleaning Up a Messy Construct. Review of Educational Research, 62 (3), 307-332.

Rodríguez Izquierdo, R. M. (2010). Éxito académico de los estudiantes inmigrantes. Factores de riesgo y de protección. Educación XX1, 13 (1), 101123.

Sales Ciges, A. (2006). La formación inicial del profesorado ante la diversidad: una propuesta metodológica para el 
nuevo espacio europeo de educación superior. Revista Interuniversitaria de Formación del Profesorado, 20 (3), 201217.

Shulman, L. S. (2005). Conocimiento y enseñanza: fundamentos de la nueva reforma. Profesorado, Revista de currículum y formación del profesorado, 9 (2), 1-30.

Taylor, C. (1993). El multiculturalismo y la política del reconocimiento. México: FCE

Tedesco, J.C. (2001). Profesionalización y capacitación docente [en línea]. Disponible en: www.iipe-buenosaires.org.ar [consulta 2005, 12 de febrero].
Thorndike, R. M. (1997). Measurenment and evaluation in psichology and education (6 ${ }^{\mathrm{a}}$ ed.). New York: McMillan.

Van der Schaaf, M. F., Stokking, K. M. \& Verloop, N. (2008). Teacher Beliefs and Teacher Behaviour in Portfolio Assessment. Teaching and Teacher Education: An International Journal of Research and Studies, 24 (7), 1691-1704.

Zeichner, K.M. (2009). Changing the epistemology of teacher education. Teacher Education and Practice, 22(4), 488-491. 


\title{
PALABRAS CLAVE
}

Educación superior, formación del profesorado, creencias, diversidad cultural.

\section{KEY WORDS}

Higher education, teacher training, beliefs, diversity (cultural).

\section{PERFIL ACADÉMICO Y PROFESIONAL DE LAS AUTORAS}

$\mathrm{D}^{\mathrm{a}}$. María del Carmen López López, es profesora titular de de Granada y doctora en Pedagogía. Sus líneas preferentes de investigación se centran en formación inicial y desarrollo profesional de los docentes, prácticum, educación intercultural y didáctica universitaria. Algunas de sus últimas publicaciones han versado sobre formación intercultural del profesorado, evaluación de los procesos de enseñanza-aprendizaje en la universidad, formación práctica de los estudiantes y formación inicial del profesorado.

$\mathrm{D}^{\mathrm{a}}$. Eva Fca. Hinojosa Pareja, es licenciada en Pedagogía y posee Diploma de Estudios Avanzados por de Granada. Actualmente desarrolla su labor profesional en el Dpto. Didáctica y Organización Escolar de de Granada con una beca de Formación de Profesorado Universitario del Ministerio de Educación. Algunas líneas de investigación preferentes son: formación del profesorado, prácticum, educación intercultural. Tiene distintas publicaciones en revistas, libros y actas de congresos sobre estas temáticas.

\author{
Dirección de contacto: Dra. $\mathrm{D}^{\mathrm{a}}$. M $\mathrm{M}^{\mathrm{a}}$ del Carmen López López \\ Facultad de Ciencias de la Educación \\ Departamento de Didáctica y Organización \\ Escolar \\ Campus Universitario de Cartuja, s/n \\ 18071, Granada. \\ E-mail: mclopez@ugr.es \\ Dña. Eva Fca. Hinojosa Pareja \\ Facultad de Ciencias de la Educación \\ Departamento de Didáctica y Organización \\ Escolar \\ Campus Universitario de Cartuja, s/n \\ 18071, Granada. \\ E-mail: ehinojosa@ugr.es
}


Fecha Recepción del Artículo: 28. Mayo. 2010

Fecha Revisión del Artículo: 28. Octubre. 2010

Fecha Aceptación del Artículo: 30. Octubre. 2010

Fecha de Revisión para publicación: 23. Mayo. 2011 